\title{
Design of a Noninvasive Bladder Urinary Volume Monitoring System Based on Bio-Impedance
}

\author{
Rihui Li, Jinwu Gao, Hongbin Wang, Qing Jiang* \\ School of Engineering, Sun Yat-sen University, Guangzhou, China \\ Email: *lirihui@mail2.sysu.edu.cn \\ Received July 2013
}

\begin{abstract}
For the needs of bladder urinary volume noninvasive monitoring in clinical, we present a noninvasive bladder urinary volume monitoring system based on bio-impedance. The system uses a four-electrode structure, which is composed of a pair of excitation electrodes and a pair of measurement electrodes. The Direct Digital Frequency Synthesis (DDS) is applied to generate a $50 \mathrm{kHz}$ sine current excitation source. The impedance information extracted from phase sensibility demodulation technology is transferred to a computer through Zigbee wireless technology for real-time monitoring. Two experiments are taken to verify the accuracy and feasibility of the system. The experiments results show that the system can accurately measure the corresponding electrical impedance change of the bladder. The system provides a new way to continuously and noninvasively monitor the bladder urinary volume of patients with bladder dysfunction.
\end{abstract}

Keywords: Bio-Impedance; Bladder Urinary Volume; DDS; Zigbee

\section{Introduction}

It is normal that bladder fullness causes an urge to void. However, for some handicapped with spinal cord injury or some patients with urological disease, this sense will disappear, causing urinary tract infections and urinary reflux even ultimately lead to renal failure. As a result, more and more patients are requiring for professional nursing, leading to the increase of the intensity of the work staff and the patient's medical costs. It is necessary to find a way to automatically monitor patients' bladder urinary volume and help them to void.

In clinic, draining urine out by a catheter inserted in the bladder is the traditional method to solve this problem [1]. But this method is invasive and may cause urinary tract infection. Another way to deal with this problem is to measure bladder urinary volume by using ultrasound. After Holmes firstly measured the bladder urinary volume by ultrasound in 1967, some ultrasound instruments such as B-mode ultrasound measurement and real time 3D ultrasound measurement were applied to bladder volume measurement [2-4] in an imaging way. But the structure of ultrasound is relatively complex and expensive. Although some special handheld ultrasound bladder urinary volume measurement devices have been developed in these years [5], all these devices are expensive and unable to measure the bladder urinary volume continuously.

\footnotetext{
${ }^{*}$ Corresponding author.
}

Bioelectrical impedance technique is also called medical electrical impedance. It extracts biomedical information related to human's physiological and pathological conditions based on the electrical characteristics and variation of biological tissues and organs. It usually applies a small AC current or voltage to the detection object by using an electrode system and detected the corresponding electrical impedance changes, from which we can access to the relevant physiological and pathological information. Long time non-invasive monitoring, high detection sensitivity, rich information of function, low cost and easy to use, are always the main advantages of bio-impedance technology [6].

The conductivity of the bladder urine is changing during natural bladder filling. Talibi et al. [7,8] have proved that bladder urinary volume and electrical impedance are closely related. The study of Liao et al. [9] has proved that bladder urinary volume can be measured by detecting the corresponding electrical impedance changes of bladder.

In this paper, a noninvasive bladder urinary volume monitoring system based on bio-impedance has been implemented. This system, uses a four-electrode structure and a $50 \mathrm{kHz}$ sine current excitation source, measures the impedance information of bladder real-time with simple operation. We have tested the accuracy of the system and the ability to measure the bladder urinary volume to make sure that the circuit structure and measurement method is feasible. 


\section{System Design}

\subsection{The Measuring Principle}

Usually, the impedance characteristics of biological tissue can be represented by the three components equivalent model [10], shown in Figure 1. $R_{i}, R_{e}$, Cm represent the equivalent biological tissue inner and outer resistance and membrane capacitance.

Biological tissue shows different impedance characteristics in different frequency, which can be represented by the Cole-Cole circle diagram [11], shown in Figure 2. $\mathrm{Z}_{\mathrm{i}}$ and $\mathrm{Z}_{\mathrm{r}}$ respectively represents the real and imaginary part of impedance, while $Z$ and $\theta$ respectively represent the modulus and phase angle of impedance. Rigaud et al [12] has proved that the impedance characteristics of biological tissue are rich in the range of $10 \mathrm{kHz}$ to 300 $\mathrm{kHz}$. Therefore, the system uses a $50 \mathrm{kHz}$ sine signal as the excitation source.

In this paper, the system uses a four-electrode structure which is composed of a pair of excitation electrodes and a pair of measurement electrodes. A $50 \mathrm{kHz}$ sine voltage signal is generated by a Direct Digital Frequency Synthesis chip (DDS, AD9833, Analog Device Instrument). The $50 \mathrm{kHz}$ voltage signal is converted to a $50 \mathrm{kHz}$ current signal through a circuit called voltage controlled current source (VCCS). The $1 \mathrm{~mA}$ amplitude and $50 \mathrm{kHz}$ sine wave current signal is fed into the bladder through the excitation electrodes. The instrumentation amplifier (AD620, Analog Device Instrument) is selected to constitute the front stage amplifier for extracting the small differential voltage signal that detected by the measurement electrodes. Then the amplitude of impedance extracted by a phase sensibility demodulation chip (AD8302, Analog Device Instrument) is transferred to a computer through Zigbee wireless technology for real-time monitoring.

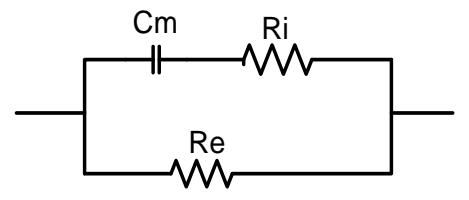

Figure 1. The three components equivalent model.

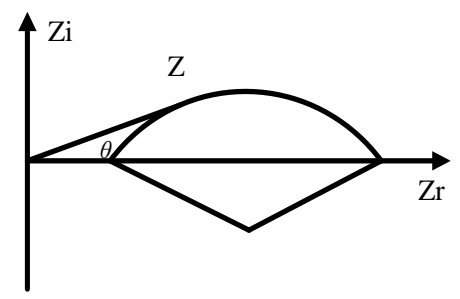

Figure 2. Bio-impedance response of the tissue in different frequency.

\subsection{System Hardware and Software}

The system is composed of two parts, including the data acquisition module and the human-computer interaction module. The data acquisition module is responsible for measuring and transmitting the impedance of the bladder while the human-computer interaction module realizes the real-time display and monitoring of Bladder impedance information as well as data storage function. These two modules are communicating through the Zigbee wireless network technology. The whole system block diagram is shown in Figure 3.

The data acquisition module is mainly composed of three parts.

a) The MCU (CC2530, Texas Instrument)

CC2530 is the core of the data acquisition module with an integrated RF wireless transceiver and a 14-bit high accuracy A/D converter, which can effectively meet the need of hardware design and simplify the circuit.

b) The excitation channel

DDS, VCCS and some peripheral components form the excitation channel. Firstly, the DDS chip AD9833, which could directly produce sine wave current with constant amplitude $\left(600 \mathrm{mV}_{\mathrm{p}-\mathrm{p}}\right)$ and adjustable frequency (0 - $12.5 \mathrm{MHz}$ ), is programmed to generate a $50 \mathrm{kHz}$ sine voltage signal. Equation (1) shows how to control the frequency we want.

$$
f_{\text {out }}=\mathrm{M}^{*}\left(f_{M C L K} / 2^{\wedge} 28-1\right)
$$

where $f_{M C L K}$ is the digital clock input of AD9833 with a $25 \mathrm{MHz}$ input from a crystal oscillator, $\mathrm{M}$ is frequency control word, which we can set it to the range of [0, $\left.2^{\wedge} 28-1\right]$ to generate an output frequency from 0 to $12.5 \mathrm{MHz}$. Then the sine wave voltage signal with $50 \mathrm{kHz}$ frequency is fed into a filtered by a high-pass filter (HPF) whose cut-off frequency is $2 \mathrm{~Hz}$ to filter the components of DC and low-frequency. Finally, the circuit called voltage controlled current source (VCCS) converts the sine wave voltage signal to a $1 \mathrm{~mA}$ amplitude and $50 \mathrm{kHz}$ sine wave current signal. The circuit of VCCS is designed with two general operational amplifiers (AD620

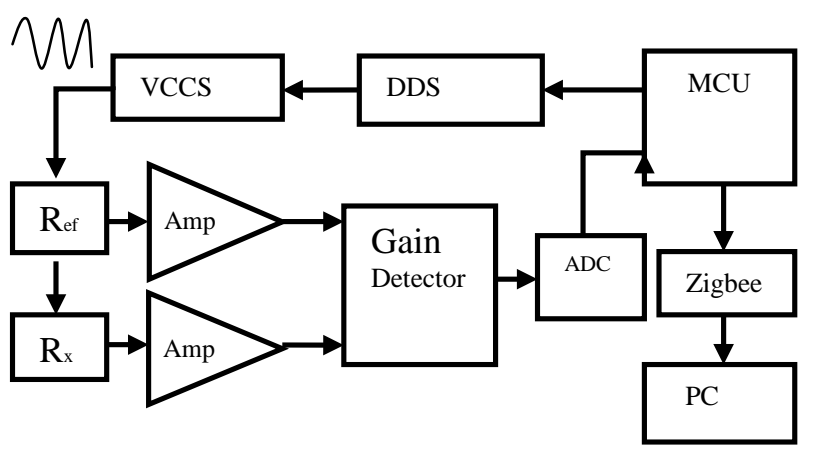

Figure 3. Block diagram of the system. Rx represents the impedance of bladder, while Ref is a reference resistor. 
and AD817, Analog Device Instrument), which have low noise, low drift and high precision characteristics, to achieve much stable sine wave current. Then the sine wave current signal is fed into the bladder $(\mathrm{Rx})$ and a reference resistor (Ref) through a pair of excitation electrodes.

c) The measurement channel

It mainly consists of differential amplifier circuits, phase sensitive demodulation circuit and a 14-bit high accuracy A/D conversion circuit. Firstly, two sets of tiny voltage signals, one is picked up from the bladder and another is picked up from the reference resistor, are respectively sent to the Pin1 and Pin2 of the AD8302 after the front stage differential amplifier (AD620) amplification process. The function of AD8302 is calculating the difference of amplitude between the two sets of voltage signals. In the frequency less than $1 \mathrm{MHz}$, the difference can be calculated by Equation (2).

$$
V_{M A G}=R_{F} L_{S L P} \log \left(V_{1} / V_{2}\right)+V_{C P}
$$

where $V_{1}$ and $V_{2}$ are respectively the input voltage of the Pin1 and Pin2, $V_{M A G}$ is the output corresponding to the magnitude of the signal level difference, $R_{F} L_{S L P}$ is 600 $\mathrm{mV} /$ decade or $30 \mathrm{mV} / \mathrm{dB}$ with a center-point of $900 \mathrm{mV}$ $\left(V_{C P}\right)$ for $0 \mathrm{~dB}$ gain. A range of $-30 \mathrm{~dB}$ to $+30 \mathrm{~dB}$ covers the full-scale swing from $0 \mathrm{~V}$ to $1.8 \mathrm{~V}$.

$V_{M A G}$ is fed into the MCU (CC2530) for A/D conversion with a 14-bit high accuracy, using an external $1.8 \mathrm{~V}$ voltage provided by AD8302 as a reference voltage of $\mathrm{A} / \mathrm{D}$ conversion. After the $\mathrm{A} / \mathrm{D}$ conversion, the impedance of bladder can be figured out by the MCU according to $V_{M A G}$ and the reference resistor. Finally, impedance data is transmitted to the human-computer interaction module through the Zigbee wireless transmission function which is integrated into the CC2530. Figure 4 shows the prototype of the data acquisition module with a portable design.

The human-computer interaction module consists of two parts: the data receiving module and the monitoring

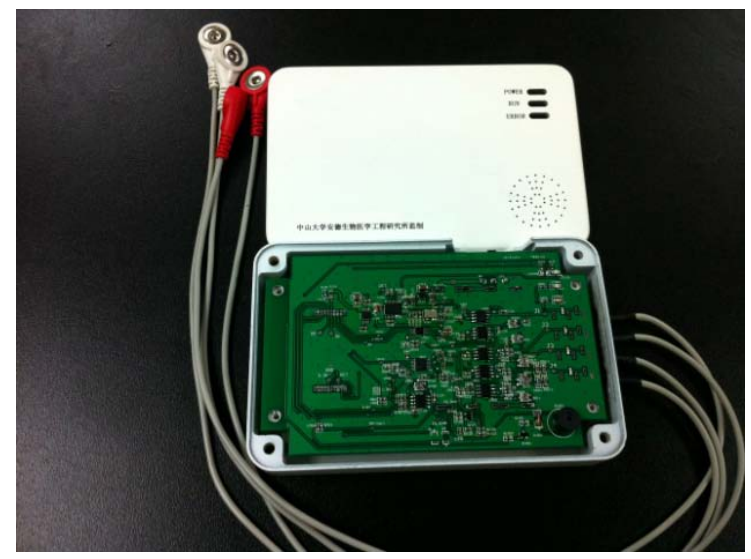

Figure 4. The photograph of the data acquisition module. software. The data receiving module, which is inserted into the computer with a USB interface, could receive the impedance data from the data acquisition module through the Zigbee wireless technology and transmit the data to the computer monitoring software. A MCU (CC2531, Texas Instrument) is selected as the core of the data receiving module, which is integrated with Zigbee wireless transmission function. The monitoring software is developed based on Labview. It provides the operators with a good human-machine interface which we can observe some impedance information such as how the impedance changes by the increasing volume of bladder. We can also save the measurement results for subsequent research and analysis.

\section{Experiments and Results}

\subsection{Accuracy of Measurement}

As the paper mentioned above, the impedance of the human bladder shows a downward trend as the increase of bladder urinary volume [9]. According to previous laboratory experiments, the impedance values of human bladder are generally in the range of $10 \Omega-30 \Omega$ and the impedance variation of the human bladder may be less than $1 \Omega$ from the beginning of accumulation of urine to urinating. Therefore, the ability to measure a related small amount of impedance change needs to be verified. In this experiment, a variable resistance is selected. The accuracy of it is $1 \%$ and the resistance value is $50 \Omega$. The excitation electrodes of the system are respectively connected to each end of the resistor. The measurement electrodes are also respectively connected to each end of the resistor, shown in Figure 5. "A+" and "A-" represent the excitation electrodes, "V+" and "V-" represent the measurement electrodes.

Frequency of the exciting current is $50 \mathrm{kHz}$ and peak-to-peak value is about $1 \mathrm{~mA}$. From $10 \Omega$ to $30 \Omega$, we use the system to measure and record every value of the variable resistance with an increment of $0.5 \Omega$. The Agilent E4980A Precision LCR meter with accuracy of $0.05 \%$ is used to calibrate the variable resistance and confirm that each time our adjustment is accurate. Then,

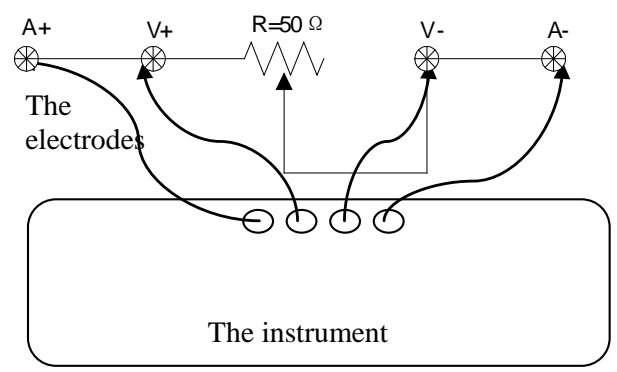

Figure 5. The connection of accuracy measurement experiment. 
we calculate the interval between each two adjacent values that we have measured by using the system, the results are shown in Figure 6.

Figure 6 shows that there is a strong positive correlation between the reference and the measurement data. Therefore, the system is able to measure a small amount of impedance changes.

\subsection{Human Experiment}

In this experiment, a healthy male college student is selected to participate in the bladder urinary volume monitoring experiment. Before the experiment, the subject is drained of urine. Then he drinks $100 \mathrm{ml}$ of water and lies in bed without any movement. The excitation electrodes and the measurement electrodes are attached to the surface of subject' bladder, the location is shown in Figure 7. The bladder impedance of the subject is monitored and recorded by the system until he feels a strong need to urinate. The variation of the bladder impedance the subject is shown in Figure 8.

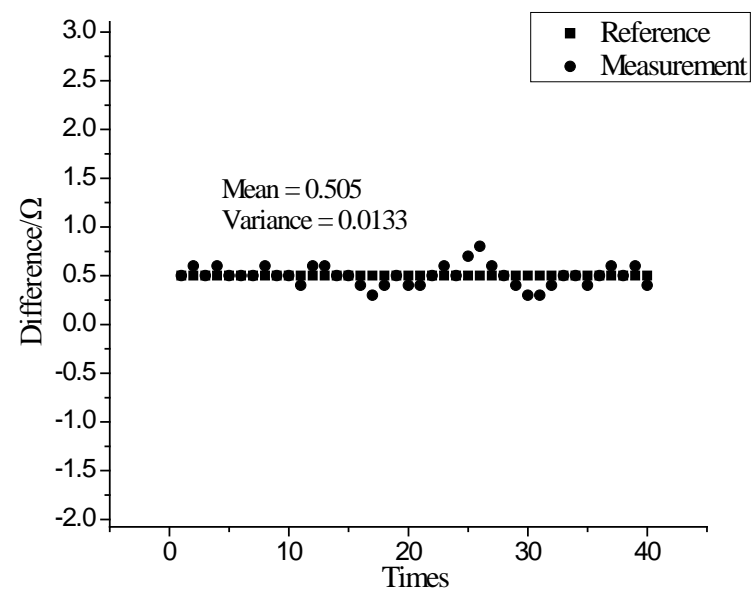

Figure 6. The mean and variance of the measurement intervals compare to the reference interval $(0.5 \Omega)$.

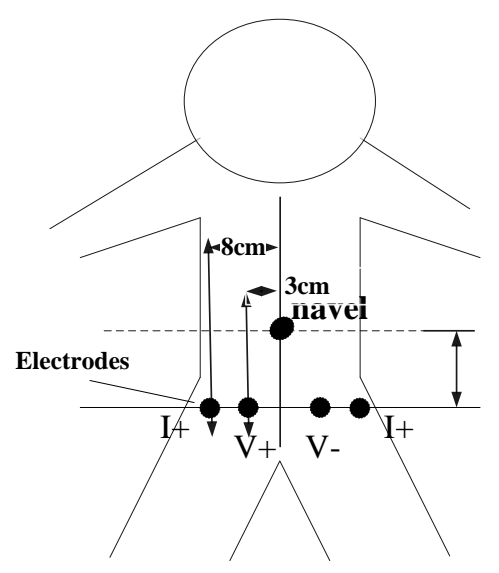

Figure 7. The location of the electrodes.

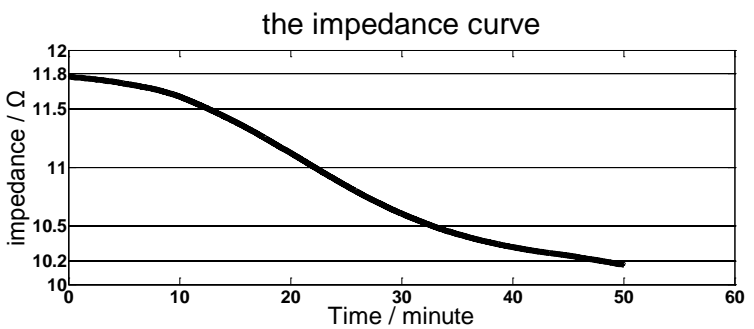

Figure 8. The impedance curve as the bladder urinary volume is accumulating.

Figure 8 shows that the system is able to monitor the changes of the patient's urinary volume by measuring variation of the electrical impedance of their bladders.

\section{Conclusions}

In this work, a high-precision and noninvasive bio-impedance system for bladder urinary volume real-time monitoring is designed and built. The system, which is integrated with some advanced and mature technologies such as Bio-impedance, DDS, Phase-sensitivity demodulation and Zigbee, has advantages on detecting sensitivity, long time non-invasive monitoring and extracting weak signals from human body effectively.

Its performances are appreciated in the preliminary experiments by showing its great ability to monitor the changes of the subjects' bladder urinary volume through measuring variation of the electrical impedance of their bladders. The system may be a new way to continuously and noninvasively monitor the bladder urinary volume of patients with bladder dysfunction.

\section{Acknowledgements}

The work is supported by the Guangdong province's Key laboratory of construction project-sensor technology and biomedical instrument, China (2011A060901013)

\section{REFERENCES}

[1] J. W. Warren, "Catheter-Associated Urinary Tract Infections," Infectious Disease Clinics of North America, Vol. 11, No. 3, 1997, pp. 609-622. http://dx.doi.org/10.1016/S0891-5520(05)70376-7

[2] J. H. Holmes, "Ultrasonic Studies of the Bladder," The Journal of Urology, Vol. 97, No. 4, 1967, pp. 654-663.

[3] J. Y. Hwang, et al., "Novel Algorithm for Improving Accuracy of Ultrasound Measurement of Residual Urine Volume According to Bladder Shape,” Urology, Vol. 64, No. 5, 2004, pp. 887-891. http://dx.doi.org/10.1016/j.urology.2004.06.054

[4] Y. K. Huang, Y. H. Jing, Z. Zheng and Y. Ran, “Three Dimension Ultrasound Research Platform Based on Virtual Instrument and Clinical Application," Journal of Electronic Measurement and Instrument, Vol. 22, No. Z1, 
2008, p. S2.

[5] H. J. Niu, et al., "Design of an Ultrasound Bladder Volume Measurement and Alarm System," 5th International Conference on Bioinformatics and Biomedical Engineering, Wuhan, 10-12 May 2011, pp. 1-4.

[6] O. G. Martinsen and G. Sverre, "Bioimpedance and bioelectricity basics,” Access Online via Elsevier, 2011.

[7] M. A. Talibi, et al., "A Model for Studying the Electrical Stimulation of the Urinary Bladder of Dogs," British Journal of Urology, Vol. 42, No. 1, 1970, pp. 56-65. http://dx.doi.org/10.1111/j.1464-410X.1970.tb11908.x

[8] F. M. Waltz, G. W. Timm and W. E. Bradley, "Bladder Volume Sensing by Resistance Measurement," IEEE Transactions on Biomedical Engineering, Vol. 1, No. 1, 1971, pp. 42-46.

http://dx.doi.org/10.1109/TBME.1971.4502788
[9] W. C. Liao and F. S. Jaw, "Noninvasive Electrical Impedance Analysis to Measure Human Urinary Bladder Volume," Journal of Obstetrics and Gynaecology Research, Vol. 37, No. 8, 2011, pp. 1071-1075. http://dx.doi.org/10.1111/j.1447-0756.2010.01487.x

[10] R. E. N. Chao-shi, "Electrical Bioimpedance Measurement Technology,” China Medical Device Information, Vol. 10, No. 1, 2004, pp. 21-25.

[11] K. S. Cole and R. H. Cole, "Dispersion and Absorption in Dielectrics I. Alternating Current Characteristics,” The Journal of Chemical Physics, Vol. 9, No. 4, 1941, pp. 341. http://dx.doi.org/10.1063/1.1750906

[12] Rigaud, B., et al., "In vitro Tissue Characterization and Modeling Using Electrical Impedance Measurements in the $100 \mathrm{~Hz}-10 \mathrm{MHz}$ Frequency Range,” Physiological Measurement, Vol. 16, No. 3A, 1995, p. A15. 